\title{
Altfranzös. dh (ð) \\ in altenglischen und altdeutschen Lehnworten.
}

\section{I.}

Ueber engl. faith und andere frz. Lehnworte des Englischen, die ein seltsames th aufweisen, hat sich seit etwa I5 Jahren eine kleine Litteratur ${ }^{1}$ angesammelt. Man findet aber das Belegmaterial nirgends beisammen, und ich glaube meinerseits dasselbe vermehren zu können, indem ich besonders - wie schon in Gröbers Grundrifs der Roman. Philol. I, 397 - auch Eigennamenmaterial verwerte. Ich würde aber darauf jetzt nicht zurückkommen, wenn die Erscheinung nicht für eine weit ältere Zeit einige Bedeutung hätte. Es lassen sich nämlich sowohl im Angls. wie im Altsächs. und Ahd. Spuren dieses selben $d h(\delta)$ in solchem Umfange nachweisen, dafs dadurch die Existenz eines afrz. $d h(\succsim)$ auch schon für das 8. Jahrhundert erwiesen wird.

Bei weitem die meisten Belege liefert die Oxforder Laudhandschrift der Sachsenchronik. Abgesehen von dem einen appellativen nativité, das zu den Jahren I I02. I 105. I I06. I I08. I I I3. I I I4. I115. I1 6 begegnet für "Weihnachten", sind es nur Eigennamen. Um die gleiche Zeit begegnen für König Louis Loðewís I I08. I I 16. I I 24 (an der letzten Stelle liest Earle gegen die Handschrift Lodewis) und die frz. Stadt Caen lat. Cadomum, die I070 als Kadum auftritt, wird 1086. I 105 Caðum genannt, und so lesen wir auch zweimal in einem von Zupitza Anglia I, 196 mitgeteilten selbständigen Chronikfragment für die Jahre III3-1114. So ist Rouen lat. Rotomagus I I 24 Roðem (= an. Ruðu) und Mantes lat. Medante 1086 Maðante, Gerberoi bei Beauvais 1079 Gerbornéð. Aulser diesen Ortsnamen treffen wir noch Lothringen, das I 126 als Loherenge erscheint, 1080 als Hloðeringa. Vereinzelt finden wir A內elís 1121 , Godefreið I I 23. I I30, Gosfreiठ I I 27 neben Gosfrei I096, Rotbert Peccéd 1123 für Robert Peche. - Den Abt Vitalis, der 1076 Vithele heifst, nennt eine andere Chronikhandschrift 1077 Fipele.

1 Vgl. bes. Varnhagen AfdA 9, 179 ZfRomPhil. 10, 298; ferner Nicol Academy 18, 581 ; Roeth, Ueber den Ausfall des $d$ im Normann. S. 21 ; Mall Computus Einl. S. 87; Koschwitz z. d. ält. frz. Denkm. S. 30; tBrink Chaucer Gramm. 70; Behrens in den Frz. Stud. V Heft 2 S. 176 (Pauls Grdr. I, 83I); Pogatscher QF 64, S. 107. Aus diesen Arbeiten wie aus den me. Wörterbüchern stammt ein Teil der unten beigebrachten Belege. 
Alle diese Chronikeintragungen, denen wir diese grammatischen Materialien entnehmen, sind kaum beträchtlich später gemacht worden als die betreffenden Jahresangaben anzeigen. Dabei bleibt zunächst auffällig, dals der Eigenname, der in Liebermanns Anglonorm. Geschichtsquellen so oft als Radulfus erscheint (s. 5. 14. 27. 47. 53. 77. 78 u. s. w.) im Laudms. der Sachsenchronik als Raulf I I 14. I I I5. I I 22 auftritt; es war der Erzbischof von Canterbury (Radulf Razulf Angl. I, 196). Und schon 1055. 1075 treffen wir einen Raulf eorl (1076 Rawulf in einer andern Hs. bei Earle S. 213). Und wir sahen oben, dafs neben Gosfreid 1127 Gosfrei I096 steht. So geht das Verstummen des Lautes frühzeitig neben den ursprünglichen Formen nebenher. Aber der eigentliche Zeitraum dieser $\partial$ war das I I. Jahrhundert und der Anfang des 12. Jahrhunderts für das französische auf engl. Boden und um $1100 \mathrm{mag}$ das Verklingen des $\delta$ um sich gegriffen haben. Hieraus ergiebt sich für einige erst um 1200-1300 auftretenden Lehnworte des Engl. der Anhalt, dafs sie der Zeit Wilhelms des Eroberers angehören müssen.

Chronologisch ist das sonstige Belegmaterial allerdings nicht von so abgrenzender Beweiskraft. Es sind nur wenige Worte, in denen sich das anglofrz. $\delta$ zeigt, und wenn man von den schott. assyth, bountith, daintith und poortith als unsicher $\mathrm{zu}$ beurteilen absieht - sie sind, von dainteth abgesehen, erst spät bezeugt, zufrühst assyth bei Barbour und bountith in den York Mysteries cf. Murray NED. s. v. - , so hat nur das einzige ne. faith eine gesicherte Existenz gehabt, während alle sonst in Frage kommenden Worte im späteren Me. kaum noch bezeugt sind. So hat das nativiteঠ der Sachsenchronik bisher keinen me. Beleg gefunden; in der Ancr.Rule 412 gilt dafür schon nativite. Bei Orrm (um 1200) treffen wir caritép karitép vv. 3000. 3008. IOII7. 10120 und das gilt auch noch in den etwa gleichzeitigen Vices and Virtues S. 19. 21.47. 63. 67, welcher Text aber auch carilé 37. 39. 45 und charite 39. $4 \mathrm{I}$ aufweist - und charite ist herrschend in der Ancr.-Rule 2. 8. 30. 1 24. 224. 408 (auch OEH. p. 57, Ayenb. 79, Gen.-Exod. v. 1016); daneben cherite Hom. I, 39. 63. 69. I 39 und cariled Chro. II 37. Zu herrschendem plenté (Ancr.-R. 194; Havel. v. I 729; Ayenb. I61) findet sich plentéd Gen.-Exod. v. 3709.

Neben dainté (Ancr.-R. 412) gilt daintéth nach Mätzner in mannigfachen Schreibungen noch Seven Sag. 606 (dayenteth), Town.Myst. S. 245 (dayntethe), Destr. of Troy 463 (deintithe), Ant. of Arth. Str. 14. 15 (dayntethis). Für maugré (Havel. I I 28; Ayenb. 69) findet sich maugreth 1 bei Hazlitt Remains I, 17 I, Leg. of the Holy Rood II I v. 125 und P. Plowman ed. Skeat. $\mathrm{Zu}$ me. druerie $=$ afrz. druerie "Liebe" gilt Wohunge of ure Loverd I, 269 druð "Ge-

1 Beachtenswert sind, worauf Varnhagen hingewiesen hat, die Schreibungen maugrêf All. Poems II, 44. 54. Gawain 1565; Alexander (Skeat) 1747. 2782; vgl. den Reim charite : lef Hom. I, 57 (Pater noster v. 4I). 
liebter". Bei feith faith treffen wir Schwanken mit einem gleichberechtigten fai; vgl. für feith Gen.-Exod. 2 I 87. 2678; Havel. 2852 (aber fei Havel. 255. I666) - feith ( feiz $b$ ) Will. 275. 828. 858; Rob.-Gl. 1551.1

Im 14. Jahrhundert gilt me. assẹth(e) "satisfaction" aus afrz. asseth; Belege Hamp. Pr.-Consc. 36ro. 3746; Wyclif I. König. 3, 14; I. Ezdra 5, 5 ; Markus I 5, 1 5; Chaucer, Rom. of the Rose 5602; Wyclif, Sel.-Works II, 237 ; Langland, P.-Plowm. B XVII, 237; Rel. Pieces p. 6. Halliw., Dict.

Vereinzelt findet sich neben ademant - aymont auch athemaunt bei Chaucer, Knight's Tale I I32. - Das schwierige me. dapeit dahét mag hier erwähnt werden mit einem Hinweis auf die Erörterung im NED. über das sicher frz. Lehnwort.

Das me. Belegmaterial ist hiermit noch nicht erschöpft. Der Name David, der im Angls. als Dawid (Dawid nur Sweet OET. 3 I 3. 4I5) und im ME. als David erscheint, zeigt im frühen Me. sehr oft die Form Davíð. Zahlreiche Belege dafür aus Orrm s. im Glossar von White und Holt II, 564 b; aufserdem noch St. Margerete ed. Cockaigne S. 18 (varia lectio); Hickes III, 170. I7I; Vices and Virtues S. 3I. 59. 8I. I I 5; Haly Meidenh. S. 9. I I. 35? 39; Juliana S. 62. 63. OE. Hom. I, 91. 97. 273. II, 31. 33. 89. 91; Wicliff Math. I, 6. 17. Der frz. Charakter dieses $\delta$ äufsert sich auch darin, dafs das Wort auch mit verklungenem $\delta$ als Davi im ME. auftritt z. B. St. Margarete ed. Cockaigne S. I 8 (mit der Variante Davið, Morris' OE. Miscel. S. 231 (im Reim auf tenderlí); vgl. die Beteuerungsformel bi Seint Davi (im Reim auf Grimesbi) Havel. 2867; Davi auch in der Digbyhs. v. Harrowing of Hell v. 195. 201) u.s.w. u.s.w. (aber auch Sein David Rob. of Glouc. 3980. 9484 etc. - Sein Davit 3977. 9179). Der frz. Charakter dieses Bibelnamens ${ }^{2}$ verträgt sich wohl mit der Thatsache, die durch Lehnworte wie me.pręche = frz. prêcher dokumentiert wird, dafs auch das kirchliche Leben Englands seit der Eroberung unter frz. Einflüssen stand. Es bleibt uns noch eine frühme. Lautform für "Jude" anzuführen, das gewöhnlich als ğ qu ğéw (ne. Jew) = afr. Giu auftritt; nur bei Orrm II, 189. 211 treffen wir eine $\delta$-Form Jupẹ́wess (dafür Orrm I, 76. II, I I9. 173 Judẹwess und in der Sachsenchonik a. 1137 Judeus). ${ }^{3}$

1 Schüddekopf, Sprache u. Dialekt des Gedichtes Wilh. of Pal. S. 45 und andre nehmen an, faith stehe unter dem Einfluls einer Analogie von engl. Abstrakten wie wealth, length, strength, health; dagegen ist zu bemerken, dals eine solche Annahme für die Sprache des 13./14. Jahrhunderts absolut unmöglich ist; denn feith ist immer einsilbig, welthe lengthe strencthe helthe immer zweisilbig.

2 Gehört hierher Orrms $N \delta p=N o e$ ? und Abiü $p=$ Abias?

8 Es scheint mir bisher nicht beachtet zu sein, dafs mit der normann. Invasion der ganze Sprachgebrauch der Kirche französiert wurde. Dafür zeugt das anlautende $d \check{z}$ in $\mathcal{F}$ ssus (ae. sagte man übrigens nur $C r t s t$, nie $\mathcal{F}$ esus), Fohdn (Fohọn $=$ Fón, ne. Fohn; ae. sagte man nur Fohannes). Fos $\$$. Facob; hierber gehört auch die Formel flum forddn. Im Orrmulum zeigt zěrrsalám (gespr. jersalફ̧m) den richtigen angls. $\zeta=j$-Anlaut, wie er in angls. Zeit 
Dieses anglofranzös. Juð̣u, das im 12 . Jahrhundert gelebt hat, führt uns $\mathrm{zu}$ einer Thatsache, die sich kurz so formulieren lärst: Das Wort Judaeus hatte schon im 9. Jahrhundert auf german. Boden eine Form mit innerem $\partial$. Der Heliand nämlich zeigt in der Cottonianischen Handschrift ${ }^{1}$ mehr als fünfzigmal eine Form Juðeo, die aus germ.-asächs. Sprachregeln nicht zu erklären ist und für die Kögel AfdA. 19, 228 Anm. auch keine Erklärung kennt. Schon Kögel bestätigt die $\partial$-Form durch einen Hinweis auf afries. Jotha und ich erinnere noch an die angls. Runeninschrift, die auf dem jetzt im Britischen Museum befindlichen Kästchen von Walrofsbein steht - Giupeas (Sweet OET S. 127). Alle diese zerstreuten Zeugnisse scheinen mir in ihrer gegenseitigen Unabhängigkeit so wichtig, dafs ich ahd. Judeo Plur. Judeon, das nie mit innerem $t$ auftritt, lieber zu asächs. Juðeo als $\mathrm{zu}$ asächs. Judeo stellen möchte. Dann sollte man allerdings wohl eine Schreibung Judheo in alten Texten noch vereinzelt antreffen, so z. B. im Isidor, wo sich oft inneres intervokalisches $d h$ - allerdings schon dafür $d$ - zeigt, aber nur Judeo schreibt. So genügt denn altsächs. Juðeo auch ohne das althochdeutsche Judeo eine $\delta$-Aussprache für Judaeus in den dem deutschen Sprachgebiet benachbarten frz.-roman. Gebiet etwa um $800 \mathrm{zu}$ erweisen. $\mathrm{Zu}$ afries. Jotha, das für den Nachbardialekt mitbeweist, stimmt ein anderes fries. Zeugnis: dem ahd. vogat entspricht nach v. Helten Altostfries. Gramm. S. 97 im Afries. öfter fogeth; das aus fries. Lautregeln nach v. Helten nicht deutbare th st ein vogầ $=$ advocatus.

So beruhen ahd. sîda "Seide“ und krîda „Kreide" auch wohl auf $\partial$-Formen, für die wir allerdings keine graphische Gewähr irgendwoher beibringen können. Und zwar charakterisieren sich diese Formen als relativ jung durch die Vertretung von lat. $\hat{\varepsilon}$ durch $\hat{\imath}$ : wären lat. crêta sêta etwa gleichzeitig mit bêta (ahd. biazza) und mensa (ahd. mias) aufgenommen, so wären ahd. *kriazza *siazza, älter vor der zweiten Lautverschiebung krẹta sệta zu erwarten. Daher gehen ahd. krîda sîda auf mutmafsliche Grundformen krệঠa sẹða zurück.

auch für Fohannes facobus u. s. w. gegolten hat. Hierher gehört me. Eǵypte $=$ ne. Egypt. Vom Frz. beeinflurst sind auch me. Fariséu "Pharisäer", Saducệu "Saducäer"; Filippe „Philippus“, Herode „Herodes", Nicodẹm „Nicodemus", auch Ma $e_{e} u$ "Matbäus", Andrẹ "Andreas". Für Ostern erscheint (frz.) Păske. Ich hoffe in kurzer Zeit das frz. Lehnmaterial des Me. einer erneuten Prüfung zu unterziehen und werde dann auf dies Problem zurückkommen.

1 Die Belege für $7 u ð e o$ mit Spirans sind einschliefslich der von Kögel für die Formel $\mathscr{f} u \partial e o$ liudi beigebrachten zwölf Belege Hel. v. 640. 696. 766. 788. 791. 910. 1473. 2340. 4700. 4811. 4826. 4845. 4850. 4913. 4925. 4939. 4953. 4955. 5052. 5057. 5104.5109 .5112 .5127 .5133 . 5136.5150 .5154 .5176$. 5180. 5212. 5214. 5223. 5232. 5238. 5240. 5245. 5259. 5275. 5283. 5294. 5310. 5326. 5345. 5358. 5379. 5387. 5409. 5413. 5470. 5481. 5525. 5721. 5749. 5800 . Es überwiegt auch im Cottonianus die Form fudeo, die im Monacensis allein gilt. 
Dieses $\partial$ ist nämlich in der Pauls Grdr. I, 320 behandelten lat. Lehnwörterschicht, die das German. in den fünf ersten nachchristlichen Jahrhunderten aufgenommen hat, durchaus nicht anzutreffen. Die german. Zeugnisse für dieses $\partial$ stecken in Lehnworten, die sich auch durch sonstige Lautkriteria als relativ jung erweisen. Das bei Otfrid um 870 bezeugte ahd. fidula "Fiedel" beruht mit ae. fiðele deutlich auf einer gemeinsamen Grundform fioula, und der Zusammenhang mit der roman. Sippe von frz. viole beweist Uebergang von anlautendem lat. $v$ in $f$, wie er nur jüngeren Lehnworten (cf. vers aus lat. versus) zukommt: in den älteren Lehnworten ist lat. $v$ durch $w$ vertreten. Auf der Grenze zwischen diesen beiden Gruppen steht die Entlehnung von lat. paraverêdus ins Deutsche: die mhd. Form pferfrît ist par(a)frềdus mit der $f$-Aussprache des lat. $v$; cf. ahd. $p$ ferf rîd; die mhd. ahd. Nebenform $p$ ferît aber scheint für $p$ ferirît $=\not$ ferizerîd mit Verlust von $w$ vor $r$ zu stehen. Uns ist hier wichtig, dafs dem Worte hd. $d$ zukommt und dals wir diesem $d$, da die zweite Lautverschiebung den Anlaut betroffen hat, ein $\delta$ (paravrềঠo) zu Grunde legen müssen. Graff III, 347 kennt eine andd. Glosse pereth $=$ hd. pferîd. Das $\hat{\imath}$ entspricht dem von ahd. sîda krîda. - So erscheint tappêtum im Ahd. als teppîth teppî̀, Graff 5,348 aus tappệdo.

Dieses th ist durch ae. fiðele dokumentiert. Noch in einem Falle wird es durch die Uebereinstimmung mehrerer Dialekte erwiesen. $\mathrm{Zu}$ den christlichen Lehnworten gehört lat. synodus $=$ ahd. sënod (sënoth AdGl. I, 247), afries. senath, ae. sinot.l Zwar ist es nicht unmöglich, dafs die Endung dieser Formen unter dem Einflufs eines german. Suffixes -ôpus steht, aber notwendig ist die Annahme in Bezug auf das th nicht. Noch ist an die geläufigen ae. Pflanzennamen celeðonie lat. chelidonia und saðerige lat. satureia $\mathrm{zu}$ erinnern, auf die Bugge hingewiesen hat. Vereinzelt abbodessa Chron. E a. 680 .

Das ae. Material lärst sich aus kontinentalen Eigennamen vermehren. Wichtig ist, weil früh überliefert, Cundop in zwei Handschriften der Sachsenchronik a. 883. 884? aus $\operatorname{com}(i)$ tatum $=$ frz. Conde. Etwa gleichzeitig mit dem letzten Beleg steht in der Chronik a. 885 Juðitle (Jupytte) für Judith. In einer jüngeren Hs. der Chronik a. 887 erscheint der langobardische König Guido als Wiঠa.

Unsicher ist anord. markaðr „Markt“ gezen me. market zu beurteilen; denn sein $\partial$ könnte auch frz. $d$ vertreten, wie anord. $R u \partial u$ "Rouen" auch keine $d$-Form voraussetzen mufs. Unsicher ist natürlich auch die Beurteilung von frz. Lehnworten des Ndl., weil mndl. $d$ (im Auslaut durch $t$ vertreten) sowohl $d$ wie $\delta$ repräsentiert: mndl. citeit "Stadt", planteit, prioreit „Priorat, Kloster", fosseit „Graben", triniteit, magesteit u. s. w. können ebenso gut auf $\delta$-Formen zurückgeführt werden wie auch nach Malsgabe der frühme. Judeus

1 Thurneysen erinnert für th an bret. creiz „Kreide“ aus einem afrz. creiðe Idg. Forschgn. IV Anzeiger S. 45. 
Chro. 1 137, cariled ib. I1 37, Kadum (Caðum) ib. 1070 gedeutet werden; und mndl. plainlet „Fülle" kann sich zu früh me. plenté $\delta$ stellen.

\section{F. KLUGE.}

II.

Die intervokalischen Gutturalen des Latein. sind in den germanisch-romanischen Ortsnamen des gallischen Grenzlandes noch zu Anfang des 5. Jh. unverändert übernommen, acu ist ach, Breisach, Andernach, Kreutznach, iacu $>$ ich, Zülpich, Morzig, Jülich, (Aquae $>$ Aachen), magu $>$ magen, Dormagen, Neumagen, Remagen, Warmagen. Dem entspricht es, dafs die germanischen Verschlufslaute in Nordfrankreich die Entwicklung der lateinischen zeigen, beschränkter die Gutturalen, bei welchen vielleicht früh besondere Assimilationserscheinungen sich geltend machten, in gröfstem Umfang Dentale und Labiale, in ungefähr allen altfränkischen Entlehnungen. Die bretonischen Einwanderer im 5.-6. Jh. übernahmen, wie Loth, Rom. Jahresber. I, 266 feststellt, noch die lat. $c, t, p$. Das Latein des 7. Jh. bietet dann reichliche Belege des Wandels zu $g, d, b$. Dals die lat. Media älter aspiriert war, wird für $b$ ziemlich einstimmig angenommen, erklärt bei $g$ die verschiedene Entwicklung von agu und acu (Dao- f. Dago- seit 652), und wird demnach auch bei $d$ der Fall gewesen sein: ${ }^{1}$ die Schreibtradition hielt an $d$ fest, während fränk. th (entsprechend $c h$ für $h$ ) im 5. Jh. mit Verständnis angenommen und noch lange neben $t$ unverstanden nachgeschrieben wurde. Im Endergebnis ist ursprünglich $p$ und $b \mathrm{zu} v$ geworden, bzw. geschwunden, $t$ und $d$ geschwunden. Dafs dem Ausfall der Dentalen ein $d h$ vorausgegangen sei, wurde zuerst von Paris, Alexius S. 96 ausgesprochen, und ist jetzt, der physiologischen Wahrscheinlichkeit und der anglonormannischen Schreibung th gegenüber, so ziemlich allgemeine Ansicht. Den Eintritt dieser Aspiration hat man sich indessen, wenigstens so weit gedruckte Aeufserungen vorliegen, viel zu spät gedacht, im II. Jh. (s. z. B. Meyer-Lübke, R. Gr. I, 363), obwohl die Strafsburger Eide intervokalisch durchaus $d h$ bieten in aiudha (zweimal), cadhuna und den Namen (aber podir). Koschwitz, Commentar 31 neigt dazu, hier einen Laut zu sehen, wie es Nicol, Academy 18, 173 that, lärst aber dann doch die Möglichkeit zu, dals nur der fränkische Schreiber eine Eigentümlichkeit seiner Sprache rein graphisch übertragen nabe. Wie seitsam, dafs dieser $d$ und $d h$ im Deutschen, aber nicht im Franz. zu trennen weifs, dafs er nur bei französischen, nicht

1 Suchier schrieb Grundrifs I, 581 : „Dals im Französischen und Mittelrhonischen zuerst die Erweichung der stimmlosen Laute erfolgte, und dafs dann mit den primären $g d b$ auch die sekundären, aus $p t c$ entstandenen, zu Reibelauten fortschritten". Dem gegenüber kann auf die Gründe für vglat. $b>v$ hier nicht eingegangen werden; franz. widerspricht central $a b a>0 u e, a p a>$ eve, clavu $>$ clou, capu $>$ chief. Für acu gegen agu mag auf Acutciacun $>$ Aiguisy, Acunun $>$ Notre Dame d'Aygu gegen Agusta $>$ Aouste, Agustodunum $>$ Autun hingewiesen sein. 
auch bei lateinischen Worten den Fehler macht und genau da macht, wo später der Laut fällt, dafs er auslautend franz. $d$ und $t$ früh zu unterscheiden vermag! $\mathrm{Da} p>v$ gegeben ist (savir), wäre Aspiration wahrscheinlich, auch wenn sie nicht dastünde. Es kann hier nur eine absichtliche Lautunterscheidung vorliegen. Graphisch allerdings ist sie auffällig, und es verlohnt sich sie einmal in dem gebotenen Zusammenhang zu betrachten, dem mit Nithards Schrift überhaupt.

Das Zeichen $d h$ kann nicht aus dem Gallofränkischen stammen, das nur th kannte; es ist auf französischem Boden völlig vereinzelt. Auch th war schon im 7. Jh. unverstanden; im St. Legier wird es (vgl. Rom. I, 286) ohne klare Absicht gesetzt, eher unter dem Eindruck, dafs es den Doppellaut meine, und im 12. Jh. ist es rein englisch. Die hochdeutschen Denkmäler (s. Braune, Ahd. Gramm. 2. A. S. I 36) haben das dem 8. - 9. Jh. angehörige lautliche Zwischenglied nur teilweise geschrieben; die deutschen Eide haben intervokalisch $d h$ ebenso wie die französischen und wie überhaupt die deutschen und französischen Eigennamen in den vier Büchern Geschichten trotz der im übrigen latinisierten Form. Der Kopist, der uns das Buch erhalten hat, war ein Romane, nach verschiedenen Anzeigen; ${ }^{1}$ nach dem Zustand der Ueberlieferung in den drei Sprachen liegt $z$ wischen ihm und dem Original, wenn überhaupt ein Zwischenglied, schwerlich mehr als eines. Er übernimmt die $d h$ da, wo sie ihm am auffälligsten waren, in den Eiden, den ca. 180 Lodhuuicus und Lodharius (einmal Ludovicus, einmal Lotharius). Dabei zeigt viermal in den Eiden, dreimal im übrigen Text (II, 9 . IV, 3. 4) meist nachträglich korrigiertes $h$ für $d h$, wie seltsam ihm im Grund die Bezeichnung war. Bei den isolierteren Personennamen versagt zum Teil seine Aufmerksamkeit; der achtmal genannte Adhelhardus hat noch fünfmal $d h$ gegen dreimal $d$, zwei Adhelbertus, ein Madhelgaudus stehen gegen zwei Adelgarius, zwei Fludualdus, ein Fredericus, ein Rodulfus, während unverstandenes edhilingi bleibt. Ebenso bei den Ortsnamen: Cadhellonensis, Cadhellonica (Calalaunis Chalons) steht dreimal, Cadelonensis einmal, ein Nordhunuuig, dagegen je ein Laudunensis, Rodanus, Spedonna. Es ist mit Sicherheit anzunehmen, dafs im Original hier überall $d h$ stand, wenn auch nicht in den anders gearteten Madasconis, Milidunensis, Viridunensis (gegen werdhan, wirdhit), Bituricas, Rotomacensis. Fehlschreibung, als welche man das Zeugnis für den franz. Laut hat abstreiten wollen, ist nirgend eingetreten, germ. $d$ bleibt in viermaligem Vodo, in Hildigardis, Hilduinus u. a. Ausschliefslichem $d$ für germ. th in den -baldus, -fridus, -nidus (neun Fälle) entspricht deutsch eid, got, frz. quid, d.h. im Auslaut wird $d$ geschrieben; im Anlaut steht $t$ für unverschobenes th, nicht vom Kopisten, da in diesem Punkt der

1 Nach Alciodorensis für Autisiodorensis geradezu ein Gallier, wahrscheinlich ebenda, wo im 15. Jh. die Handschrift sich befand, in St. Magloire zu Paris, 
westliche Einflufs über den Rhein herüberreicht, vgl. Braune l. c. I39 Anm. 9; in Kompositen vor Konsonant th: Mathfridus, Nithardus.

Nithards Muttersprache war diejenige Karls d. Gr., die hochdeutsche. Er verbrachte seine Jugend in der Villa bei der Aachener Pfalz, wo Angilbert etwas nach 800 die Söhnlein durch sein poetisches Sendschreiben begrülsen lälst, lebte dort jedenfalls, ebenso wie Berta, bis zum Todesjahr seines Vaters und des Kaisers, und wird, nach dem relativen Alter Angilberts und Bertas zu rechnen, gegen Ende des 8. Jh. geboren sein. Der spätere Wohnort mulste wesentlich von den Besitzungen abhängen, jene der Berta lagen vorzugsweise auf französischem Gebiet (Simson, Jahrb. Ludwigs d. Frommen I, 18), die des Sohnes ebenda, wie mit Bestimmtheit aus seiner politischen Stellung im Bruderkampf zu erschliefsen ist. Hier mufste er notwendig auch das Französische erlernen. Nithard war der Gesandte Karls an Lothar, der offizielle Verfechter seiner Politik, der lateinischen und der beiden Vulgärsprachen mächtig: in den Stralsburger Heerlagern fand sich kaum ein anderer, der, so wie er, befähigt gewesen wäre die Schwüre zu formulieren. Ihre Aufnahme in den lateinischen Bericht bleibt ungewöhnlich und auffallend (Misc. Caix S. 83), auch wenn man mit Rajna Rom. XXI, 53 die momentane politische Bedeutung des Wortlauts betont; sie ist natürlich, wenn sie von dem Berichterstatter abgefafst waren. Im lateinischen, deutschen und französischen Text ist eine deutsch eigenartige, französisch ganz isolierte Orthographie durchgeführt: wenn Nithard die Eide nur kopierte, wäre diese schwerlich in das Deutsche, sicher nicht in das von ihm sonst kaum geschriebene Französisch eingedrungen. Nithard ist also der Verfasser der Eide.

Die scheinbare Ausnahme von der Schreibregel, welche viermaliges fradre bietet, während die Annahme gleichlaufender Entwicklung von $d r$ mit intervokalischem $d$ durch das oben von Kluge erbrachte ags. saeperige (das mittlere $e$ ist ags.), entsprechend franz. sarriette, bestätigt wird, ist leicht $\mathrm{zu}$ erklären. Nithard kannte deutsch $d r$, nicht $d h r$, es ist also natürlich, dals er es nicht schreibt, auch wenn er es sprechen gelernt hatte, und auf dialektische Erhaltung kann daraus keinenfalls geschlossen werden. Ueberhaupt gestattet die Bestimmung des Verfassers keinen Rückschlufs auf den franz. Dialckt, da wir nicht wissen, wo N. sein Französisch gelernt hat. Eine gewisse Wahrscheinlichkeit spricht für die Picardie; er selbst ist zwar, wenn überhaupt, erst am Schlufs seines Lebens Laienabt von St. Riquier geworden (s. Wattenbach, Geschichtsquellen 6. Aufl. I, 2 I4), aber sein Vater verwaltete neben der Abtei den ducalus totius maritimae terrae, und Hariulfs Biographie versteht darunter (vgl. dort III, 9) die dem Kloster nahe liegenden Küsten. An eine derartige Stellung pflegten sich auch Erwerbungen zu knüpfen. Diese aber können auch das Erbe Hartnids geworden sein. Berta war bei Noyon und Angers begütert, der durch königliche Vergabung erlangte Besitz pflegte nach Zufall zerstreut zu sein. 
Selbst das südliche Burgund ist nicht ganz ausgeschlossen, obwohl dies wesentlich zu Lothar hielt. Die Beurteilung wird noch schwieriger als sie bisher erschien: Nithard mochte mit einer germanisch und lateinisch gefärbten persönlichen Sprache um so eher auskommen, als noch keine Region und keine Stadt einen sprachlichen Vorrang beanspruchen durfte. Wir haben Germanismen in der Sprache selbst, nicht nur solche des Schreibers (vgl. zuletzt Schwan, Zts. f. r. Ph. XI, 464) zu befürchten. Ein positives Resultat ergiebt sich indessen eben aus dieser Erwägung: vol, poblo, pois meinen $o$, nicht $u o$, da der Deutsche dieses sprach und schrieb, daher auch franz. gesprochen und geschrieben haben würde, selbst bei leichter Differenz des Klanges und bei abweichendem Accent. Ob freilich $q$ noch nicht diphthongiert war oder uo nach Labial zu $\circ$ reduziert ist, bleibt dahingestellt. Ebenso kommen zu franz. dreit die deutschen nohhein, eid, leistit und warnen, savir, podir, ${ }^{1}$ sit als unvollkommene Schreibungen zu erklären. Mit den andersartigen $i$ für $e$ setzt $N$. nicht die umgekehrten Schreibungen des Merowingerlateins fort, denn sein Latein kommt aus der Karlsschule, aber französisch steht er unter analoger Einwirkung, in, ind, ist erzeugt prindrai: vom Deutschen aus hätten ihm drei verschiedene $e$ oder mindestens zwei (da $\bar{e}$ bald darauf $e a$ ist) zur Verfügung gestanden, $e<a$ und $\ddot{e}$. Die $u$ für $o$ in amur, dunat (nunquam ist von vorne bis hinten lateinisch) lassen eher auf wirkliche Qualität schliefsen, da der Druck des Lateinischen bei diesem Laut erheblich schwächer ist als bei e. Die Eigennamen heifsen lateinisch Lodharius und Lodhuuicus, 2 letzteres entschieden traditionell gegen die allgemeine deutsche Aussprache, deutsch Ludher und Ludhuwig, französisch gekreuzt und bedeutungslos Ludher und Lodhuvig; zu beachten ist französisches Fludualdus II, 10, III, 3. Ziemlich bedeutungslos ist bei einem Eingewanderten, der vom Latein aus Französisch gelernt hat, die von Meyer Zts. XII, 526 hervorgehobene Erhaltung des Endvokals in poblo, nostro gegenüber fradre; sendra und fradra beweisen, dais einfach vokalisches $r$, bzw. vokalisches $l$ gemeint ist.

Während primär oder sekundär gedehnte $t$ und $d$ (soudain) sich erhalten, fällt die inlautende und auslautende einfache Dentale, nach Suchier (Reimpredigt XXI ff., Grundrifs 581 ) in der ersten Hälfte des 12. Jh., während andere den Prozels schon im ausgehenden II. beginnen lassen, so Paris Rom. XI, 4OI und Extraits ${ }^{4}$ 5, Neumann Zts. XIV, 563. Dals die Normannen 1066 den Inlaut noch sprachen, erhellt schon aus der immerhin beträchtlichen Ausdehnung, in welcher sich die angelsächsische Schreibung

1 Deuten dorthin, wo veir, seir zu Hause ist.

2 Das deutsche Ludwig setzt eine Form hluthu, nicht hlotha voraus, Ludher ist mhd. ebenso Luther; die Vorliebe für $i$ und $u$ im deutschen Text, von welcher Schwan Zts. XI, 464 spricht, kann ich dort nicht finden. Die merowingischen Autoren und Urkunden haben unverkennbar Chlotacharius etc. mit Tenuis neben Chlodoveus etc. mit Media. Eines der vielen Namenrätsel. 
th hier festsetzen und bis zur Mitte des 12. Jh. erhalten konnte (Alexius, Brandan, die Glossen Jhb. XVII, 43, Cambridger Psalter, im Oxf. Ps. zwei th, im Computus Arundel zwei th, ein $\delta ; p$ oder $\delta$ in den Aelfrikglossen Zts. X, 297); entsprechend ist in den Glossen Raschis, der I IO5 starb, das $d$ erhalten (Rom. I, I 56), wobei freilich die Einbeziehung noch etwas älteren Materials zu berücksichtigen ist. Einmaliges cruel im St. Legier nebst korrigiertem Oste[d]un wird noch nicht als Zeichen des beginnenden Ausfalls zu betrachten sein; im Domesdaybook ist er so häufig - mehr als das Doppelte der Zts. VIII, 360 verzeichneten Fälle wäre anzumerken - dafs er als Lautregel $\mathrm{zu}$ betrachten ist; regelmälsig dort auch Rolland, einmal Roric. Entsprechend lälst um $1200^{1}$ der festländische südwestliche oder südliche Schreiber des Hohenliedes ebenso oft $d$ weg als er es einsetzt, bei vollständiger Verwirrung im Auslaut; auch für ihn ist bei Berücksichtigung des starken Drucks der Ueberlieferung, unter welcher er stand, völliges Verstummen anzunehmen. Die direkt oder indirekt in den Beginn des 12. Jh. zurückreichende anglonormannische Schreibertradition wie die Reime Philipps de Thaun (Mall, Computus S. 80) berechtigen zum selben Schluls: die $d$ wie die th sind antiquarische Ware, die höchstens in der Kirche noch halb lautete. Es zeigt sich das auch darin, dafs (in den von Roeth, Hall. Diss. 82, zusammengestellten wie in den von ihm nicht einbezogenen Quellen) sich keinerlei Einwirkung der Umgebung auf den Ausfall feststellen läfst. Obwohl diese gerade bei den Dentalen eine schwache ist, dürfte die Artikulation sich zuerst zwischen identischen oder fast identischen Vokalen verschliffen haben, wie in espedhe, medhesme: und so rasch der weitere Verfall vor sich gegangen sein mag, würde das bei Schreibern, die im Anfang der Bewegung stünden, noch zu bemerken sein. Damit berührt sich im Südosten frare, crollet, escueyr, espaa des Alexanderfragments, noch etwas südlicher in einer Urkunde aus dem Ende des II. Jh. Aam, Oalric, civaa etc. (aber Peire, Freirics), s. Meyer, Alexandre II, 84 und 89. I I 66 hat der Ausfall die Normandie noch nicht erreicht, um 1200 ist er überall vollzogen, und er muls, bei der gewaltigen Ausdehnung, im Rückland der Normandie bedeutend früher begonnen haben, wahrscheinlich noch vor der Mitte des II. Jh.

Auslautende Dentalis (abgesehen von den proklitischen Wörtchen) besteht in den frühesten Denkmälern. Doch hat neben arde tost, das durch den identischen Anlaut genügend erklärt ist, die Eulalia perdesse $s a$, und da für den Schreiber $z$ und $s$ zweierlei sind, liegt hier entweder ein Anzeichen des Ausfalls vor Kons. überhaupt vor, und zwar gerade vor einem Konsonanten, bei dem die Aussprache nicht die geringste Schwierigkeit machte, oder ein Schreibfehler. In der Jonaspredigt ist die Lesung cherte in Z. 29

1 P. Meyer und neustens Schum (Rom. Jhsb. I, 675) stellen ihn noch ins II. Jh., andere (vgl. Koschwitz, Kommentar S. 170) ins erste Viertel des 12. 
nach dem Zustand der Zeile, wie sie die Photographie zeigt, ganz unsicher zu nennen; $f u c o$ in $32^{r}$ ist ziemlich deutlich, würde sich aber erklären wie oben arde tost, $t+t s=6$. Sicher ist $15 \mathrm{fu}$ seche, dabei aber zu beachten, dals dem Geistlichen auch sonst noch einzelne Buchstaben unter den Tisch gefallen sind. Der provenzalische Schreiber des St. Legier hat in seiner Vorlage das ihm fremde fud jedenfalls vorgefunden; im Lambspringer Alexius ist der Auslaut noch ausnahmslos geschrieben. Im Anglonormannischen bieten eine Anzahl von Schreibungen mit th Brandan, Cambridger Psalter, auch zwei die A-Hs. des Computus, eine S und eine die Aelfrikglossen: keine der Alexius gegenüber 40 inlautenden. Im Domesdaybook meint th nur $t$, ebenso wie $c h=c$ steht. Elision bei unbetontem -et findet sich im Rolandslied in 20 unter 60 Fällen (cf. Rom. III, 399), während sie der Karlsreise wahrscheinlich ganz fremd ist. Zahlreiche umgekehrte Schreibungen im Hohenlied sichern Abfall auch unter dem Ton und nach Konsonant, daneben steht dort metrisch mindestens Hiat. Das Domesdayb. verhält sich gegen Abwurf im Auslaut entschieden ablehnend (unbet. -et fehlt), einige sichere - $n$ für -nd mögen dorther stammen, wo $z \mathrm{u} n d>n$ vor Verschlufslaut auch noch $n d>n$ vor $r$ hinzukam, begünstigt durch die flexivische Analogie Colebranz - Colebran = anz - an, arpenz - arpen. Ueber das Verhältnis des einfachen Auslauts zum Ton und zum folgenden Anlaut geben die Schreiber keine Auskunft, -th sowohl als $-d$ und $-t$ erscheinen von der persönlichen Tradition bestimmt. Der vollständige Mangel auslautender th im Alexius spricht für Verschiedenheit vom Inlaut und vergleicht sich mit dem Verhalten Nithards, wie mit dem des Domesdaybook; die Tendenz auf Erhaltung des Hiats in Philipps Computus (10 gegen 3) neben seiner sicheren Aussprache $-\ell$ und $-i$, steht in nur unwesentlichem Gegensatz zur stärkeren Elision im Roland und dem sicher noch ins II. Jh. zu setzenden Gormond et Isembart: besonders die beiden letztgenannten verhindern einen erheblichen Zeitunterschied zwischen dem Schwund von Inlaut und Auslaut anzunehmen und drängen damit auf die Annahme eines unmittelbaren Zusammenhanges hin, ebenso wie die Identität der Bezeichnung in den obgenannten anglonormannischen Handschriften.

Mall, Computus $8 \mathrm{I}$ ff. schlors, dafs überall $-t$ anzunehmen sei, das zuerst nach $e$ und $i$, dann nach $a$, zuletzt nach $u$ gefallen wäre; -th hielt er vom Inlaut übertragen. Suchier, Reimpredigt XIX stellt zu der von Mall nur berührten, nicht berücksichtigten Thatsache, dafs dreit nicht mit feid reimt, die andere, dafs feid und feit, aber nicht dreid geschrieben wird, und kommt zu dem Ergebnis, dals amatu $>$ amado $>$ amed geworden, während lat. -t geblieben sei; es fiel zuerst $-d$, dann -it, fut, wahrscheinlich an letzter Stelle -at. Die Sonderstellung des lat. -t wird durch Reime wie dit: partit begründet, die Schreibungen oïd etc. seien inkorrekt. Die Fragestellung ist absichtlich auf die Stimmhaftigkeit beschränkt, von der Bedeutung der th abgesehen, jene nach der Ursache schliefs- 
lich unzweifelhafter Scheidung von $d u t$ und $f u$ nicht berührt. Paris, Extraits de la Ch. de Rol. S. 15 der ersten, S. 16 der vierten Auf. fordert für das Gedicht $-d$ vor stimmhaftem Laut, $-t$ vor stimmlosem und in pausa, gesprochen ungefähr wie engl. $t h$, gegenüber -t appruý. Unbetont -et war zum Teil gefallen, $-d$ fiel später, dann unter etwas anderen Bedingungen nicht ganz gleichzeitig $t$. . Sein gestütztes $-t$ ist nach S. I 2 zu beurteilen, wo nur $r r$ als Doppelkonsonant zugelassen wird. Es sind das in den Zwischenräumen je eines Dezenniums in fortschreitender, wenn auch im einzelnen nicht einwandfreier, doch jeweilig auf der Höhe stehender Formulierung die Auffassungen derjenigen, die sich am ernstlichsten mit dem Gegenstand beschäftigt hatten, bei Mall und Suchier unter Mitteilung des seitdem nicht wesentlich bereicherten Apparats, während Paris, entsprechend dem $Z_{\text {weck }}$ seines Schulbüchleins, nur Endergebnisse ausspricht; von seinem Alexius S. 97 und 271 datiert die nähere Untersuchung, und schon dort S. 98 kam dabei auch die vom Sanskrit her alt bekannte, trotzdem nur sehr langsam begriffene Satzphonetik wenigstens für ad - habet erstmalig zur Erwägung. Für den speziellen festen Punkt, das Rolandslied, kann ich seine Anschauungen nicht teilen. Zwischen unbetont -et oder -eth und der Elision von - $e$ vor Vokal stand Hiat, notwendig in derselben, möglich auch in mehreren Generationen. Wo Elision eintritt, ist unbetont $-t$ nur mehr traditionell, nach dem thatsächlichen Gebrauch nicht einmal Hiatzeichen. Die starke Elision bezeichnet entschieden einen vorgeschrittenen Standpunkt, der zumal im Vergleich mit dem Verhalten des Computus durchgängigen Schwund im Ausund Inlaut annehmen läfst. Um so mehr als die Voraussetzung früheren Falls des unbetonten Lauts in der Ueberlieferung keine Stütze findet, ebenso wenig als etwa inlautend in der vorvokalisch auslautender Stellung accentlich ungefähr gleichwertigen in benedir etc.

Lateinisches - $t$ (nach meiner mehrfach ausgesprochenen Ansicht in unbetonter Stellung von der betonten Auslautssilbe her restitaiert) war in pausa unzweifelhaft tonlos, das bedeutet, da die proklitischen Wörtchen nicht in Rechnung zu ziehen sind, mehr als ein Dritteil der Rede. Dazu kommt die Stellung vor stimmlosem Anlaut. Vor stimmhaftem ist Assimilation nicht durchaus notwendig; man hört z. B., auch da wo sie vorvokalisch sonst eintritt, emphatisch was hát er gesagt neben was had er gesagt, anlautend $r$ und $l$ setzen nach Stimmloser ganz gewöhnlich stimmlos ein. Frühfranzösisch malsgebend ist hier jedenfalls $t$. $\mathrm{Da}$ sich festes $-t$ dauernd davon unterscheidet, mufs dieses, da es qualitativ nicht verschieden sein konnte, quantitativ verschieden gewesen sein, also gedehnt. Der Doppellaut war gegeben erstens vom Latein. aus, zweitens bei Reduktion eines vorausgehenden Konsonanten, einerlei ob dieser den vorausgehenden Vokal beeinflufst oder nicht, in deitt wie in soutt; inlautend $d d$ zuerst in cubitus, tepidus, dann in subitanus, capitellus. 
Einfaches inlautendes $t$ gelehrter Worte schlofs sich wahrscheinlich an. Der Doppellaut dauerte nachweislich, als $c a>k i a$ ward, da er Position bildet, und es liegt kein Beweis vor, dafs er vor dem 11. Jh. gekürzt worden sei. Die einfachste Auffassung ist vielmehr, dals dutt blieb, als fut schwand. Latein. nachtoniges $t$ der Paroxytona ist, wie Suchier richtig annahm, $d$ gewesen, ehe der Endvokal fiel, wie coude und chadel zeigen, und ergab also im Satz:

in pausa $-d$, vor stimmhafter $d$, vor stimmloser $t$. dagegen war lat. $-t$ :

in pausa $t$, vor stimmhafter $d$ und $t$, vor stimmloser $t$.

Die partielle Gleichheit in stimmhafter Stellung mufste in dieser rasch vollständige herbeiführen, weiterhin aber umgekehrt bewirken, dals in pausa durch die in gewöhnlicher Rede etwa doppelt häufigeren $-t$ die $-d$ verdrängt wurden. Die wenig zahlreichen lateinischen intervokalischen $d$ im französ. Auslaut werden sich den -tangeschlossen haben, auch wenn hier, wie oben vermutet wurde, vorfranzösisch die Aussprache $d h$ gegeben war: der Nominativ grez entsprach -atus, der Obliquus folgte.

Dafs schliefslich der Abfall der - $t$ von der Stellung vor Konsonant ausgegangen sei, wird durch die Fortdauer der gedehnten Dentalen unwahrscheinlich gemacht. Die th aber konnten sich auch nicht von der Stellung vor Vokal und etwa vor Liquiden aus verallgemeinern, da jene erheblich in der Minderzahl war. Wir müssen vielmehr annehmen, dafs die femininen -dhe die Maskulinformen beeinflufsten, trotz der Gegenwirkung des Nominativs, und dals von hier aus der Auslaut überhaupt ins Wanken geriet. Diese Einwirkung begann vor dem Schwund des intervokalischen Lautes, vollendete sich erst nach ihm: Den Andeutungen, welche uns die Schreibertradilion gewährt, entspricht am besten die Annahme, dals um 1066 -t zum Teil noch bestand, zu Ende des 11. Jh. -t und -th gefallen sind.

Die von Kluge erbrachten germanischen Belege bestätigen für den Inlaut in unwidersprechlicher Weise das Zeugnis der Eide und zeigen für die Zeit der Normanneneroberung die starke Ausdehnung von -th. Die bedeutend älteren sinoð und Cundop dagegen dürften aus der Suffixangleichung zu erklären sein; denn es ist zwar nicht unmöglich, aber doch nicht recht wahrscheinlich, dafs die -t des Alexius rein graphisch sind. Das entgegenstehende $\delta$ in *paravre $\partial o$ halte ich aus anderweitigen Gründen für byzantinisch, das französ. Wort für deutsch vermittelt.

G. BAIsT. 